\title{
Mutations in the urocanase gene UROC1 are associated with urocanic aciduria
}

\author{
C Espinós, M Pineda, D Martínez-Rubio, V Lupo, A Ormazabal, M A Vilaseca, , L \\ J M Spaapen, F Palau, R Artuch.
}

\section{Authors' affiliations:}

Carmen Espinós, Dolores Martínez-Rubio, V Lupo, Francesc Palau, Laboratory of Genetics and Molecular Medicine, Instituto de Biomedicina, Consejo Superior de Investigaciones Científicas (CSIC), and CIBER de Enfermedades Raras (CIBERER), Valencia, Spain.

Mercè Pineda, Aida Ormazabal, Maria A. Vilaseca, Rafael Artuch, Pediatric Neurology and Clinical Biochemistry Departments, Hospital Sant Joan de Déu, and CIBER de Enfermedades Raras (CIBERER), Barcelona, Spain.

Leo J. M. Spaapen, Department of Biochemical Genetics, University Hospital Maastricht, The Netherlands.

\section{Corresponding author:}

Dr. Rafael Artuch

Clinical Biochemistry Department, Hospital Sant Joan de Déu

Passeig Sant Joan de Déu, 2

08950 Esplugues, Barcelona, Spain

Telephone: + 34932806169

Fax: +34932803626

e-mail: rartuch@hsjdbcn.org

Key Words: Urocanic aciduria; urocanase gene; intermittent ataxia; mental retardation,. Wourd count: 3033 


\section{Key points}

- We investigate the underlying molecular defect in a patient who suffers from urocanic aciduria presenting with mental retardation and intermittent ataxia. The biochemical findings were consistent with a defect in urocanase.

- We found two putative mutations in the urocanase gene UROC1 that produce two amino acid substitutions, p.L70P and p.R450C. In silico predictions, protein expression studies and enzyme activity assays suggest that none of the mutations produce a fully functional enzyme. The p.L70P substitution, which probably imply the disruption of an $\alpha$-helix in the N-terminus, would alter its properties and therefore, its function. The p.R450C change would render impossible any interaction between urocanase and its substrate and would loss its enzyme activity. We therefore suggest that mutations in the UROC1 gene might be the cause of the urocanic aciduria in our patient.

- We found a partial cerebral folate deficiency in this case, that probably lead to a impaired pterin and biogenic amines status in cerebrospinal fluid. These findings might be explained by the fact that urocanase participates in the synthesis of folate metabolites through histidine catabolic pathway. However, low CSF 5MTHF concentrations have been related with several genetic and non-genetic conditions and consequently, this finding might be also considered unspecific. 


\begin{abstract}
Urocanase is an enzyme in the histidine pathway encoded by the UROC1 gene. We describe the first putative mutations, p.L70P and p.R450C, in the coding region of the UROC1 gene in a girl who suffers from urocanic aciduria presenting with mental retardation and intermittent ataxia. In silico predictions, protein expression studies and enzyme activity assays suggest that none of the mutations can produce a fully functional enzyme. The p.L70P substitution, which probably imply the disruption of an $\alpha$-helix in the N-terminus, would alter its properties and therefore, its function. The p.R450C change would render impossible any interaction between urocanase and its substrate and would loss its enzyme activity. Consequently, these studies suggest that both mutations could alter the correct activity of urocanase, which would explain the clinical and biochemical findings described in the patient.
\end{abstract}




\section{INTRODUCTION}

Three metabolic defects have been recognized in catabolism of histidine: histidase (MIM 235800), urocanase (MIM 276860) and glutamate formiminotransferase (MIM 229100) deficiencies [1,2]. Urocanase deficiency was first reported in 1971 [3], and mental retardation was described as the main neurological sign in a single case. Later on, two sisters were reported presenting mental retardation plus ataxia and dysarthria [4]. In these cases, the defect could be demonstrated by indirect analysis of some metabolites of histidine pathway in blood and urine and by the quantification of deficient urocanase activity in liver biopsies. By contrast, it has been suggested that urocanase deficiency might be a benign condition, as in some healthy cases urocanic aciduria was detected by screening analysis [1]. Interestingly, glutamate formiminotransferase deficiency, the following biochemical step in histidine catabolism, presents some neurological signs common to urocanic aciduria, and mutations explaining severe and mild phenotypes have been reported [2]. Here we describe a patient presenting urocanic aciduria, mental retardation and intermittent ataxia associated with two base-pair changes in the UROC1 gene that produce two amino acid substitutions. We argue in favor that these molecular changes may be the genetic cause of the clinical and biochemical picture in this patient.

\section{MATERIALS AND METHODS}

\section{Case report}

The patient is a female born of healthy non-consanguineous parents. She presented normal development in the first year of life and started to walk alone at 16 months, but the parents observed clumsy performance. At 4 years of age, she presented three episodes of severe ataxia that lasted 20-25 days, which arose with recurrent infections. 
These episodes resolved slowly during the next weeks. At 5 years old, she was referred for neurological evaluation to our hospital. Clinical examination showed truncal ataxia with broad base, ataxic gait, dysmetry more evident on the left arm, action tremor, dysartric speech, and normal ocular movements with slight nistagmus on horizontal gaze. Normal muscle strength with brisk tendon reflexes and moderate mental retardation were also observed. Cranial MRI and neurophysiological studies, including electroencephalogram, electroretinogram, visual evoked potentials, brainstem evoked potentials, electromyogram, nerve conduction velocities, fundus oculi and cardiological examinations, showed normal results. At 9 years of age she presented crises of severe ataxia of 20 days duration again. At that moment, she was attending normal school with a special program of integration for those with moderate mental retardation. Since then she has shown improvement in some neurological signs, such as tremor and ataxia. A second cranial MRI-S was normal. She is currently 19. She has slight tremor and nistagmus, walks with broad base and has a very clumsy performance. Cognitive evaluation shows IQ of 54 (WISC-R: verbal 57 and manipulation 60).

Routine laboratory analyses were normal including serum amino transferases, creatinine, urea and other parameters (no liver or renal dysfunction was detected). Screening for inborn errors of metabolism showed normal results, including plasma and urine amino acids (no histidine accumulation), blood lactate, sialotransferrin isoelectricfocusing, creatine deficiency syndromes, purine and pyrimidine defects and organic acidurias. Hypothyroidism and celiac disease were also ruled out. Biochemical and/or genetic screening for early onset forms of inherited ataxia showed normal results (Friedreich ataxia, ataxia with isolated vitamin E deficiency, ataxia teleangiectasia, abetalipoproteinemia, and ataxia of mitochondrial origin). 
Informed written consent was obtained for biochemical and genetic investigations, which were performed following diagnostic protocols approved by the Ethics Committee of Hospital Sant Joan de Déu (Barcelona).

\section{Biochemical analysis}

Urocanic acid in urine was analyzed by HPLC with ultraviolet and diode array detection as previously reported [5]. Blood count, serum folate, and plasma total homocysteine were determined by standard automated procedures. Cerebrospinal fluid was collected according to a standardized protocol, and 5-methyltetrahydrofolate, neopterin, biopterin and biogenic amines (5-hydroxyindoleacetic [5-HIAA] and homovanillic acids [HVA]), metabolites of serotonin and dopamine, respectively) were determined by HPLC with electrochemical and fluorescence detection, as previously reported [6,7].

\section{Genetic analysis}

Genomic DNA from the propositus and her father was obtained by standard methods from peripheral white blood cells. Mutation analysis of the UROC1 gene was performed by direct sequencing of PCR products using primers designed according to the reference sequence NM_144639 that amplify their twenty exons and intronic flanking sequences (Table 1) in an ABI Prism 3130xl autoanalyser (Applied Biosystems, Foster City, CA). We investigated in silico the biological relevance of the mutated residues. Conservation of residues was analyzed by alignment of related sequences using the program BLAST [8]. Secondary structure predictions were calculated with four different software tools (GOR, Jpred3, APSSP, and nnPredict), available at ExPASy Proteomics Server (http://www.expasy.ch). Sequence-based predictions of the phenotypic consequences of the UROC1 p.R450C mutation were proposed using the program SIFT [9]. We also predicted the effects of the mutation on the basis of the 3D structure using the quantitative rules of Wang and Moult [10], and the PolyPhen server [11]. Visualization 
of the structure and of the consequences of the UROC1 p.R450C mutation was carried out with the program Coot [12]. We used the structure 1UWK [13] (RCBS Protein Data Bank) that corresponds to the urocanase from Pseudomona putida in complex with its substrate, urocanate.

\section{Protein production and enzyme activity}

The UROC1 gene (cDNA clone MGC:135008, IMAGE: 40076086) was cloned into the expression vector pET100/D-TOPO ${ }^{\circledR}$ (Invitrogen), which allows expression of recombinant proteins with an $\mathrm{N}$-terminal tag containing the Xpress ${ }^{\mathrm{TM}}$ epitope and a 6xHistag. The enzyme was produced in Escherichia coli BL21Start ${ }^{\mathrm{TM}}$ (D3) (Invitrogen). The cells were induced with $1 \mathrm{mM}$ IPTG and were grown as previously described [13]. The pellet was resuspended in $5 \mathrm{~mL}$ of lysis buffer $(50 \mathrm{mM}$ potassium phosphate, $\mathrm{pH}$ 7.8, 400 mM NaCl, 100 mM KCl, 10\% glycerol, 0.5\% Triton X-100, 10mM imidazole) containing $1 \mathrm{mM}$ phenylmethylsulfonylfluoride (AppliChem) and sonicated. After removing the cells debris by centrifugation, the supernatant was applied to a His SpinTrap ${ }^{\mathrm{TM}}$ column (GE Healthcare) and eluted according to the manufacturer's instructions. Human UROC1 p.L70P and p.R450C missense mutations were generated by PCR with specific primers containing the nucleotide changes and mutated proteins were purified as described above for the native urocanase. The fractions containing the urocanase (native or any of the mutated variants) were identified by SDS-PAGE and the overexpressed bands of the desired proteins were confirmed by western blot using a mouse anti-His6 monoclonal antibody (Roche).

Urocanase was assayed by a previously described spectrophotometric method, in which the disappearance of urocanic acid is measured as decrease in absorbance at $277 \mathrm{~nm}$ [14]. The reaction mixture containing $0.5 \mathrm{~mL}$ of $0.2 \mathrm{M}$ potassium phosphate buffer, $\mathrm{pH}$ 7.55, $0.10 \mathrm{~mL}$ of $0.01 \mathrm{M}$ EDTA, adjusted to $\mathrm{pH}$ 8.0, $0.01 \mathrm{~mL}$ of $0.01 \mathrm{M}$ urocanic acid in 
$0.01 \mathrm{~N} \mathrm{HCl}$, and water in a total volume of $0.84 \mathrm{~mL}$, was preincubated at $37^{\circ} \mathrm{C}$ for 5 minutes. The reaction was started by adding $0.160 \mathrm{~mL}$ of enzyme solution, and the decrease in absorbance at $277 \mathrm{~nm}$ was monitored for about 15 minutes. The molar extinction coefficient of urocanate under these conditions is 18,800 [15]. One unit of enzyme is defined as the amount which causes the disappearance of $1 \mathrm{nM}$ of urocanic acid per min.

\section{RESULTS}

Urocanic aciduria was demonstrated at different ages (12, 14 and 18 years) with urocanis acid levels ranging between 158-202 mmol/mol creatinine (normal values below $10 \mathrm{mmol} / \mathrm{mol}$ creatinine). Urocanic acid excretion was normal in her father. Serum folate was at the lower limit of our reference interval $(10 \mathrm{nmol} / \mathrm{L}$ : reference values 10-26), although neither hyperhomocysteinemia nor megaloblastic anemia was observed at any point during the evolution of the disease. Cerebrospinal fluid results are reported in Table 2. 5-methyltetrahydrofolate, neopterin and biopterin deficiencies were observed together with an evident reduction in homovanillic acid and HVA/5-HIAA ratio. Other parameters determined in cerebrospinal fluid, such as glucose, proteins, cells and amino acids were normal. 
Table 1. PCR primers used for mutation screening of the UROC1 gene

\begin{tabular}{|c|c|}
\hline Exon & Primers \\
\hline 1 & $\begin{array}{l}\text { UROC_E1D: 5'-aatgagaggctggaggagg-3' } \\
\text { UROC_E1R: 5'-gtgctcactatggaccacc-3' }\end{array}$ \\
\hline 2 & $\begin{array}{l}\text { UROC_E2D: 5'- agacaaatccatttgggagg-3' } \\
\text { UROC_E2R: 5'- gtccetttgaagctgtacc-3' }\end{array}$ \\
\hline 3 & $\begin{array}{l}\text { UROC_E3D: 5'- ggctagatgctgtgctttgg-3' } \\
\text { UROC_E3R: 5'-tcgtgttctgtttctgctgg -3' }\end{array}$ \\
\hline 4 & $\begin{array}{l}\text { UROC_E4D: 5'- ctctccctgtctgcaactcc-3' } \\
\text { UROC_E4R: 5'-ccacacaatctccaatgctgg -3' }\end{array}$ \\
\hline $5+6$ & $\begin{array}{l}\text { UROC_E5-6D: 5' - ggtgctgcttctgttgacag -3', } \\
\text { UROC_E5-6R: 5' - atggctaacggggaaacacg-3' }\end{array}$ \\
\hline $7+8$ & $\begin{array}{l}\text { UROC_E7-8D: 5'- agtggctgggaggacctgg -3' } \\
\text { UROC_E7-8R: 5' - ggtgtaagtgatccgggagg-3' }\end{array}$ \\
\hline 9 & $\begin{array}{l}\text { UROC_E9D: 5'- acctacaggggatgacacc -3' } \\
\text { UROC_E9R: 5'- aaacaggcccacaccaagc-3' }\end{array}$ \\
\hline $10+11$ & $\begin{array}{l}\text { UROC_E10-11D: 5' - tgtgtctggggcacctatgc -3', } \\
\text { UROC_E10-11R: 5'- aactaggtggtggccagagc-3' }\end{array}$ \\
\hline 12 & $\begin{array}{l}\text { UROC_E12D: 5'- actgagggtgccacattggg-3' } \\
\text { UROC_E12R: 5'- aagcctcggtcaggatttgc-3' }\end{array}$ \\
\hline 13 & $\begin{array}{l}\text { UROC_E13D: 5'- aagaccttgtgcatccttcc -3' } \\
\text { UROC_E13R: 5'- gcttctccaacctaaataccc-3' }\end{array}$ \\
\hline 14 & $\begin{array}{l}\text { UROC_E14D: 5' - cacctgacttctggatgctg-3', } \\
\text { UROC_E14R: 5'- gtctctatggaggcagaagc-3' }\end{array}$ \\
\hline 15 & $\begin{array}{l}\text { UROC_E15D: 5'- ccctcaccttgtgtcctagc -3' } \\
\text { UROC_E15R: 5'-ttgggcagtcttctgacagc -3' }\end{array}$ \\
\hline 16 & $\begin{array}{l}\text { UROC_E16D: 5'- accccaggtcctgttattgc -3' } \\
\text { UROC_E16R: 5'-ggaagaggctgagggatgc }-3 \text { ' }\end{array}$ \\
\hline 17 & $\begin{array}{l}\text { UROC_E17D: 5'-cccttgtcctcagtctttacc -3' } \\
\text { UROC_E17R: 5'- cactctatgccgagcctctc -3' }\end{array}$ \\
\hline 18 & $\begin{array}{l}\text { UROC_E18D: 5'- acgactgctgctgtgagagg -3' } \\
\text { UROC_E18R: 5'- cccaaaacttccacatcagcc-3' }\end{array}$ \\
\hline 19 & $\begin{array}{l}\text { UROC_E19D: 5' - tccaaaacatgggcttctgc -3' } \\
\text { UROC_E19R: 5' - tgtggtcatgagaggaatcc-3' }\end{array}$ \\
\hline 20 & $\begin{array}{l}\text { UROC_E20D: 5'- gcccggttatgttgacatgg -3' } \\
\text { UROC_E20R: 5'- gcgagaagtgcaggtagtgc-3' }\end{array}$ \\
\hline
\end{tabular}


Table 2. Main biochemical features of the patient in cerebrospinal fluid*.

\begin{tabular}{lll}
\hline & Patient & Reference values \\
\hline 5-methyltetrahydrofolate & 36 & $42-81 \mathrm{nmol} / \mathrm{L}$ \\
Neopterin & 9 & $11-24 \mathrm{nmol} / \mathrm{L}$ \\
Biopterin & 10 & $14-36 \mathrm{nmol} / \mathrm{L}$ \\
5-hydroxyindoleacetic acid & 93 & $63-185 \mathrm{nmol} / \mathrm{L}$ \\
Homovanillic acid & 90 & $124-362 \mathrm{nmol} / \mathrm{L}$ \\
HVA/5-HIAA ratio & 0.98 & $1.50-3.50$ \\
\hline
\end{tabular}

* In the patient, cerebrospinal fluid was collected at 18 years of age. Results were compared with reference ranges established in our laboratory in a control population of similar age ( $\mathrm{n}=15$; age range: 16 to 20 years). 
Persistent urocanic aciduria and clinical data, mainly mental retardation, pointed towards a diagnosis of urocanase deficiency. Analysis of the UROC1 gene revealed that the patient harbored two nucleotide changes, c.209T $>C$ in exon 2 and c.1348C $>\mathrm{T}$ in exon 14 (Figure 1). These base-pair changes are translated in two amino acid changes, p.L70P and p.R450C, respectively. Moreover, sequencing analysis showed the neutral change c.1056C>T (p.G352G) in exon 11 previously described as a SNP, rs 34025926, (http://www.ncbi.nlm.nih.gov/SNP). The proband's father was heterozygous carrier of the p.R450C mutation (no DNA was available from the mother). Both nucleotide changes were searched in 200 chromosomes from healthy controls of Spanish ancestry by automated sequencing, and neither of them was detected, suggesting that they could be pathogenic mutations. Then, we proceeded to perform a phylogenetic analysis of the L70 and R450 residues. Leucine at position 70 was present in, besides Homo sapiens, some mammals (Canis familiaris, Equus caballus, Monodelphis domestica, Bos taurus and Macaca mulatta) and in Gallus gallus. The secondary structure predicted for the human urocanase showed that an $\alpha$-helix was always predicted from residue 63 to residue 70 .With respect to arginine 450, the alignment of the urocanase sequences revealed that R450 is extremely conserved and in fact no variation was found in more than one hundred aligned sequences from different organisms (data available on request). The p.R450C mutation was always predicted as a probably pathologic change by four software programs. In P. putida, the orthologue residue of human R450 is R362 which has capital importance for the enzymatic activity of the urocanase. Urocanate is tightly bound to urocanase by means of its carboxylate group, forming a buried saltbridge with R362 [13]. If this arginine is mutated to cysteine, the urocanase would lose this interaction and would therefore be unable to bind the urocanate (Figure 2). 
The protein expression analysis by SDS-PAGE, after sonication and previous to purification, revealed that both the native and the p.R450C urocanase were present in the supernatant and the pellet fractions (Figure 3A \& 3B). However, the p.L70P form was only detected in the pellet (Figure 3C). To optimize protein expression in bacteria, we performed experiments of the p.L70P mutant under different conditions of IPTG concentration, temperature and inducing time. In any case we could not obtain the p.L70P protein in the supernatant (Figure 3C). Thus, purification and enzymatic assays were only performed for the native protein and the p.R450C mutant. The spectrophotometric enzymatic assay showed that the native urocanase had a specific activity of approximately 70 units per mg of protein. By contrast, the p.R450C urocanase lacked of detectable activity.

\section{DISCUSSION}

We describe two nucleotide substitutions in the coding region of the UROC1 gene in a girl who suffers from urocanic aciduria, with mental retardation and intermittent ataxia as the main symptoms. The propositus is a compound heterozygote for the UROC1 p.L70P/p.R450C mutations. In silico analyses of the putative structure of human urocanase revealed that both amino acid substitutions could have a pathological effect. The L70 residue is only conserved in a few mammals and in Gallus gallus. Lower organisms such as $P$. putida lack it, making impossible to analyze the consequences of this mutation in the available structures of the urocanase (RCBS Protein Data Bank). The predicted secondary structure using the human urocanase sequence as a template however, suggested that the $\mathrm{L} 70$ residue could form part of an $\alpha$-helix, and then the change to proline may disrupt the $\alpha$-helix, possibly resulting in an alteration of the structure of the N-terminal region. The normal function of the enzyme therefore would 
be altered. We could not perform an enzyme activity assay for the p.L70P protein because this mutant protein was not obtained in the soluble fraction. The p.L70P protein was only observed in the precipitate. According to the in silico analysis, this mutation would produce a conformational change of $\alpha$-helix at the $\mathrm{N}$-terminus. The putative abnormal conformation could affect the solubility of the enzyme and the correct enzyme function. The p.R450C mutation affects an extremely conserved residue and no change could be tolerated in this position according to in silico analyses. Because of the predictable interaction by a salt-bridge between the R450 in the human urocanase and its substrate, urocanate, according to the structure of $P$. putida [13], the substitution of R450 by a cysteine would imply that urocanase could not interact with urocanate. That is why an enzyme with the p.R450C mutation would not be able to metabolize urocanate and, therefore, the enzyme would be deficient and then urocanic acid accumulates and excretes in urine in large amounts. In fact, enzyme activity was not detectable in the p.R450C urocanase purified extracts from the bacterial expression system whereas that the native urocanase had a specific activity of approximately 70 units per mg of protein. Moreover, neither of these two changes has been identified in 200 control chromosomes, providing additional support for the view that these nucleotide changes do not represent polymorphisms and may be the causative pathogenic mutations. Consequently, in silico predictions, protein studies and enzyme activity assays would suggest that none of the mutations, p.L70P and p.R450C, in urocanase could produce a fully functional enzyme.

The healthy father of the patient is a carrier in heterozygosis of the UROC1 p.R450C mutation. This finding would indicate that the urocanase deficiency is a hereditary disease. To date, four symptomatic children with urocanic aciduria have been reported: two isolated cases [3,16], and two affected sisters [4]. With regard to the two-sisters 
family previous generations were also possibly affected, which would suggest autosomal dominant inheritance. The genetic analysis performed in the propositus and in her father allows us to conclude that the urocanase deficiency seems to be a genetic disorder with autosomal recessive inheritance. The other defects of the histidine catabolism, histidase and glutamate formiminotransferase deficiencies, are also autosomal recessive disorders.

The propositus suffers from urocanic aciduria presenting mental retardation and ataxia, and also tremor and nistagmus. These latter neurological signs have not previously been associated with urocanase deficiency $[1,3,4]$. Histidine catabolism comprises several metabolic steps leading to the synthesis of 5-10-methenyltetrahydrofolate, a precursor of the purine ring and other folate metabolites. Although the biosynthesis of folate derivatives through histidine catabolism might represent a minor source of single carbon units [17], we found low-normal blood folate together with decreased CSF 5-MTHF values in our case. As previously reported for other folate metabolism disturbances $[18,19]$, we also found an association between decreased folate values in CSF, pterin deficiencies and decreased values of HVA, the main metabolite of dopamine. In this sense, tremor and probably other neurological signs might be related with dopamine deficiency. Furthermore, symptoms such as ataxia and psychomotor retardation have commonly been observed among patients with cerebral folate deficiency, even those with very slight cerebral folate deficiency [20]. However, low CSF 5-MTHF concentrations has been related with several genetic and non-genetic conditions [17, 18], and consequently, this finding might be also considered unspecific.

It has been demonstrated that patients with glutamate formiminotransferase deficiency also suffer from mental and physical retardation, which reinforces the hypothesis that a severe metabolic blockade in urocanic acid catabolism might cause the clinical 
phenotype. An explanation for differing phenotypes among patients with urocanic aciduria might be similar to that suggested for glutamate formiminotransferase deficiency [2], and factors such as severity of mutations, amount of accumulated urocanic acid, impaired central nervous system folate, pterins and dopamine status and other unknown factors might be involved. Urocanic acid has been related with skin photoprotection and regulation of immune response [21,22], although no immune alterations or skin abnormalities were observed in our case. The precise implication of urocanic acid accumulation in the clinical phenotype therefore will require further investigation.

In conclusion, we suggest that mutations in the UROC1 gene might be the cause of the urocanic aciduria in our patient. In spite of we cannot demonstrate a definite causative relationship of UROC1 mutations and the mental and neurological syndrome in this patient, we propose that mutational analysis of the urocanase gene is advisable in this rare condition, along with evaluation of folate deficiency consequences in central nervous system, such as pterin and dopamine status or other biological markers of impaired folate status.

\section{ACKNOWLEDGMENTS}

We are grateful to the patient for her kind collaboration. We are indebted to Dr. Clara Marco-Marín for in silico structural studies. The authors declare that they have no competing financial interests. This work was supported by grants from the Fondo de Investigación Sanitaria (PI051318 and PI070548). The CIBERER is an initiative of the Instituto de Salud Carlos III. 
The Corresponding Author has the right to grant on behalf of all authors and does grant on behalf of all authors, an exclusive licence (or non exclusive for government employess) on a worldwide basis to the BMJ Publishing Group Ltd to permit this article (if accepted) to be published in Journal of Medical Genetics and any other BMJPGL products and sublicences such use and exploit all subsidiary rights, as set out in our licence (http://JMG.bmj.com/misc/ifora/licenceform.shtml). 


\section{REFERENCES}

1 Levy HL, Taylor RG, McInnes RR. Disorders of histidine metabolism; in: Scriver CR BA, Sly WS, Valle D, Chuilds B, Kinzler KW, Vogelstein B ed. (ed): The metabolic and molecular bases of inherited disease. New York, 1995, p 1107.

2 Hilton JF, Christensen KE, Watkins D, Raby BA, Renaud Y, de la Luna S, Estivill X, MacKenzie RE, Hudson TJ, Rosenblatt DS. The molecular basis of glutamate formiminotransferase deficiency. Hum Mutat 2003; 22: 67-73.

3 Yoshida T, Tada K, Honda Y, Arakawa T. Urocanic aciduria: a defect in the urocanase activity in the liver of a mentally retarded. Tohoku J Exp Med 1971; 104: 305-312.

4 Kalafatic Z, Lipovac K, Jezerinac Z, Juretic D, Dumic M, Zurga B, Res L. A liver urocanase deficiency. Metabolism 1980; 29: 1013-1019.

5 Kuracka L, Kalnovicova T, Liska B, Turcani P. HPLC method for measurement of purine nucleotide degradation products in cerebrospinal fluid. Clin Chem 1996; 42: 756-760.

6 Ormazabal A, Garcia-Cazorla A, Fernandez Y, Fernandez-Alvarez E, Campistol J, Artuch R. HPLC with electrochemical and fluorescence detection procedures for the diagnosis of inborn errors of biogenic amines and pterins. $J$ Neurosci Methods 2005; 142: 153-158.

7 Ormazabal A, Garcia-Cazorla A, Perez-Duenas B, Gonzalez V, FernandezAlvarez E, Pineda M, Campistol J, Artuch R. Determination of 5methyltetrahydrofolate in cerebrospinal fluid of paediatric patients: reference values for a paediatric population. Clin Chim Acta 2006; 371: 159-162. 
8 Altschul SF, Gish W, Miller W, Myers EW, Lipman DJ. Basic local alignment search tool. J Mol Biol 1990; 215: 403-410.

$9 \quad$ Ng PC, Henikoff S. SIFT: Predicting amino acid changes that affect protein function. Nucleic Acids Res 2003; 31: 3812-3814.

10 Wang Z, Moult J. SNPs, protein structure, and disease. Hum Mutat 2001; 17: 263-270.

11 Ramensky V, Bork P, Sunyaev S. Human non-synonymous SNPs: server and survey. Nucleic Acids Res 2002; 30: 3894-3900.

12 Emsley P, Cowtan K. Coot: model-building tools for molecular graphics. Acta Crystallogr D Biol Crystallogr 2004; 60: 2126-2132.

13 Kessler D, Retey J, Schulz GE. Structure and action of urocanase. J Mol Biol 2004; 342: 183-194.

14 Chasin LA, Magasanik B. Induction and Repression of the Histidine-degrading Enzymes of Bacillus subtilis. J Biol Chem 1968; 243: 5165-5178.

15 Tabor H. Isolation and determination of histidine and related compounds; in: Colowick SP and Kaplan NO ed. (ed): Methods in enzymology. Vol III. New York: Academic Press, 1957, p. 633.

16 Van Gennip AH, Rajnherc J, De Bree PK, Wadman SK. Urocanase deficiency in a 7-year-old-boy with psychomotor retardation. Soc Study IEM, Lyon, France 1983; 6-9 Sept: 119.

17 Rosenblatt D. Inherited disorders of folate transport and metabolism; in: Scriver CR BA, Sly WS, Valle D, Chuilds B, Kinzler KW, Vogelstein B ed. (ed): The metabolic and molecular bases of inherited disease. New York, 1995, p 1107.

18 Surtees R, Heales S, Bowron A. Association of cerebrospinal fluid deficiency of 5-methyltetrahydrofolate, but not S-adenosylmethionine, with reduced 
concentrations of the acid metabolites of 5-hydroxytryptamine and dopamine. Clin Sci (Lond) 1994; 86: 697-702.

19 Ramaekers VT, Blau N. Cerebral folate deficiency. Dev Med Child Neurol 2004; 46: 843-851.

20 Ramaekers VT, Rothenberg SP, Sequeira JM, Opladen T, Blau N, Quadros EV, Selhub J. Autoantibodies to folate receptors in the cerebral folate deficiency syndrome. N Engl J Med 2005; 352: 1985-1991.

21 Anglin JH, Jr., Bever AT, Everett MA, Lamb JH. Ultraviolet-light-induced alterations in urocanic acid in vivo. Biochim Biophys Acta 1961; 53: 408-409.

22 Reeve VE, Greenoak GE, Canfield PJ, Boehm-Wilcox C, Gallagher CH. Topical urocanic acid enhances UV-induced tumour yield and malignancy in the hairless mouse. Photochem Photobiol 1989; 49: 459-464. 
Figure 1. Electrophoregrams of the two identified mutations in the UROC1 gene. (A) the c.209T $>$ C mutation and (B) the c.1348C $>$ T mutation, both in heterozygosis, in a healthy control and in the proband. Black arrows indicate the changes. (C) Alignment of the urocanase from Homo sapiens, Canis familiaris, Mus musculus and Pseudomona putida. Above, the L70 amino acid is only conserved in some mammals and in Gallus gallus and, Pseudomona putida lacks of this residue. Below, the R450 amino acid is perfectly conserved across all the analyzed species. 
Figure 2. Visualization of the effect that the p.R450C mutation causes in the urocanase. Figure shows the structure 1UWK from Pseudomona putida in complex with its substrate, urocanate, using the program Coot. On the left, the native structure. On the right, the R362 is changed to C362 and therefore, the salt/bridge between this residue and the substrate is lost. In P. putida the orthologue of the human R450 residue is the R362 amino acid. 
Figure 3. Visualization of the native urocanase and the mutated variants, p.R450C and p.L70P. The BL21Star ${ }^{\mathrm{TM}}$ (D3) cells overexpressing the native and mutated urocanase were sonicated and centrifugated obtaining its insoluble and soluble fractions. The soluble fraction was applied into a His SpinTrap ${ }^{\mathrm{TM}}$ column and two fractions were separated, retained (R.) and non-retained (N.R.). The retained fractions were eluted and contained the purified proteins. All obtained fractions were visualized by Coomassie Stain SDS-PAGE gel. The black arrows indicate the bands corresponding to (A) the native urocanase and (B) the p.R450C mutant, and the arrow head shows (C) the p.L70P mutant. Figure shows that similar amounts of purified protein were obtained for the native urocanase and the p.R450C mutant. By contrast, the p.L70P protein was only present in the pellet (insoluble fraction). " $\mathrm{M}$ " is the molecular weight marker SeeBlue ${ }^{\circledR P l u s 2}$ Pre-Stained Standard (Invitrogen). The estimated molecular weight of urocanase is aproximately $74.8 \mathrm{KDa}$. 